\title{
The sensitivity of a palm-based psychomotor vigilance task to severe sleep loss
}

\author{
Nicole Lamond, SARah M. JaY, Jillian DorRian, \\ Sally A. Ferguson, Gregory D. Roach, and Drew Dawson \\ University of South Australia, Adelaide, South Australia, Australia
}

\begin{abstract}
In this study, we evaluated the sensitivity of a 5-min personal digital assistant-psychomotor vigilance test (PDA-PVT) to severe sleep loss. Twenty-one participants completed a 10-min PVT-192 and a 5-min PDA-PVT at two hourly intervals during $62 \mathrm{~h}$ of sustained wakefulness. For both tasks, response speed and number of lapses (RTs $>500$ ) per minute significantly increased with increasing hours of wakefulness. Overall, standardized response speed scores on the 5-min PDA-PVT closely tracked those of the PVT-192; however, the PDAPVT was generally associated with more lapses/minute. Closer inspection of the data indicated that when the level of sleep loss and fatigue became more severe (i.e., Day 3), the 5-min PDA-PVT was not quite as sensitive as the 10-min PVT-192 when 2- to 10-sec foreperiods were used for both. It is likely, however, that the observed differences between the two devices was due to differences in task length. Thus, the findings provide further evidence of the validity of the 5-min PDA-PVT as a substitute for the 10-min PVT-192, particularly in circumstances in which a shorter test is required and/or the PVT-192 is not as practical.
\end{abstract}

A substantial increase in the prevalence of shiftwork in recent years (Smith, Macdonald, Folkard, \& Tucker, 1998) has meant that many employees are now exposed to higher levels of work-related fatigue and, in turn, often experience reduced alertness and performance, increased sleepiness, and greater risk of injury and accident (Dinges, 1995; Hakkanen \& Summala, 2000; Lauber \& Kayten, 1988; Mitler et al., 1988; Rosa, 1995). Following an extensive inquiry in 2000 , the Australian Federal Parliament established that fatigue was one of the major occupational health and safety issues within Australia's transport industries (House of Representatives Standing Committee on Communications, 2000). Increasingly since the release of this report, organizations within Australia have been conducting quantitative field studies to assess and, subsequently, manage the levels of fatigue associated with different work schedules (Ferguson, Dawson, \& Lamond, 2005; Lamond, Roach, Darwent, et al., 2002; Roach \& Lamond, 2003). Although there is no universal measure of fatigue, several tasks exist that are reliable assays of fatigue-related impairment in research settings. One task that has recently emerged is the Walter Reed palm-held psychomotor vigilance test (PVT), a field-portable reaction time (RT) test that was designed to emulate a commercial device (the PVT-192) widely used in both laboratory and field studies of sleep deprivation, shiftwork, and fatigue (Belenky et al., 2003; Dinges et al., 1997; Dinges \& Powell, 1985; Dorrian et al., 2006; Lamond, Darwent, \& Dawson, 2005).

This palm-based PVT provides users with a test that can be loaded onto small, inexpensive, widely available com- pact devices (personal digital assistants [PDAs]) that are easy to carry around. In contrast, the PVT-192 is often considered to be bulky and, as a specialized piece of machinery, it can be purchased only from a single supplier in the U.S. at a cost that would prohibit buying a large number of devices. As has been noted by Thorne, Johnson, Redmond, et al. (2005), other advantages of this test that the PVT-192 lacks include the fact that (1) multiple subjects can easily use the same device, if desired, yet still be distinguished by name or study code and (2) subjects can declare their handedness and button preference on the first session and, thus, this information does not need to be known in advance. Yet the PDA-PVT retains many of the features that make the PVT-192 a widely used task. It is easily performed and is minimally affected by aptitude, thereby maximizing its utility. Furthermore, since the test has a learning curve of only one to three trials, both the need for extensive, timeconsuming training sessions and masking effects due to skill acquisition are minimized.

Reducing the length of the task further increases the suitability of the PDA-PVT for field use. Thus, whereas the standard length of the PVT in laboratory studies has often been $10 \mathrm{~min}$, field studies involving the PDA-PVT have typically used a 5-min version of the task, since it is more practical for environments with time constraints (Baulk et al., 2007; Kamimori, Johnson, Thorne, \& Belenky, 2005; Killgore, Killgore, Ganesan, Krugler, \& Kamimori, 2006; Lamond, Petrilli, Dawson, \& Roach, 2006; McLellan, Kamimori, Bell, et al., 2005; McLellan, Kamimori, Voss, et al., 2005; Petrilli, Roach, Daw- 
son, \& Lamond, 2006). Although longer tests tend to be more sensitive to the effects of fatigue (Dinges \& Barone Kribbs, 1991; Johnson, 1982), studies have shown significant fatigue-related impairment when tasks of short duration have been used (Dawson \& Reid, 1997; Roach, Dawson, \& Lamond, 2006). Importantly, two recent studies designed to validate the PDA-PVT established that the 5-min PDA-PVT demonstrates a sensitivity comparable to that for the 10-min PVT-192 during a night of wakefulness (Lamond, Dawson, \& Roach, 2005; Thorne, Johnson, Redmond, et al., 2005), suggesting that it is a valid tool for assessing fatigue. Notably, due to mechanical differences between the testing devices (details in Thorne, Johnson, Redmond, et al., 2005), in both studies, differences were observed between the PDA-PVT and the PVT-192 when the raw scores were compared. Specifically, absolute response speed on the PDA-PVT was systematically slower. However, standardizing the scores removed these differences, so that performance on the 5-min PDA-PVT closely tracked that on the PVT-192 during 28-40 h of sustained wakefulness.

To date, these are the only two published validations of the palm PVT. Overall, they suggest that the 5-min PDAPVT may provide a reasonable substitute for the PVT-192, particularly in large-scale field studies that require a shorter test and a large number of compact devices (i.e., ones that are easy to transport and carry around). Notably, however, both studies involved only moderate sleep loss (i.e., one night of wakefulness); thus, the sensitivity of the PDAPVT to greater levels of sleep loss and fatigue has yet to be examined. Since there is increasing use of the PDA-PVT in field studies, such research would be beneficial. The present study aimed to further validate the 5-min version of PDA-PVT for use as a viable alternative to the 10-min PVT-192 in the field by assessing its sensitivity during 2 nights $(62 \mathrm{~h})$ of wakefulness.

\section{Method}

Subjects. Twenty-one healthy individuals ( 8 of them female, 13 male), with a mean $( \pm S D)$ age of $21.9 \pm 3.5$ years, participated in the present study. The subjects were nonsmokers who did not regularly consume large doses of caffeine or alcohol. Those recruited had no current health problems and were medication free (including over-the-counter medications), with the exception that the female subjects could continue birth control medications. All were self-reported good sleepers who did not habitually nap and had not undertaken shift work or transmeridian travel in previous months. Before the study commenced, the protocol was approved by the University of South Australia Human Research Ethics Committee, using guidelines established by the National Health and Medical Research Council of Australia. Prior to study commencement, all the subjects provided written informed consent.

Procedure. The subjects spent 4 consecutive days in-residence in the laboratory, in groups of 3 or 4 . The initial days involved an adaptation and baseline night and training. On the adaptation day, the subjects arrived at the laboratory at $1200 \mathrm{~h}$ and were assigned to their individual bedroom. After they had been provided with verbal and written descriptions of study procedures and rules, the subjects completed a training session to familiarize themselves with the performance tasks. Prior to retiring at $2300 \mathrm{~h}$, a standard montage of electrodes was applied to each subject's face and scalp. Following $9 \mathrm{~h}$ time in bed [TIB], the subjects were awakened at $0800 \mathrm{~h}$, and the electrodes were removed so that they could shower and breakfast.
The subjects then completed further training to minimize learning effects. On the baseline night, electrodes for monitoring sleep were applied, and the subjects were required to be in bed from 2300 to $0800 \mathrm{~h}$. The following day, the period of extended wakefulness (62 h) commenced.

During every second hour of wakefulness, the subjects completed a 10-min PVT-192 and a 5-min PDA-PVT. The PVT-192 and PDA-PVT are both hand-held devices, which required the subjects to attend to the display for the duration of the test. In the present study, the Zire71 hand-held device (PalmOne Inc., U.S.) was used, but other PDAs have also been successfully utilized. As quickly as possible after the appearance of a visual stimulus (for the PVT-192, the LED timer commenced counting upward in milliseconds; for the PDA-PVT, a black bull's-eye appeared on the screen), the subjects pressed the appropriate response key with the thumb of their dominant hand. During testing sessions, the subjects were seated alone in a room in front of a blank wall. To eliminate order effects, the presentation of the tasks was randomized and counterbalanced. As per standard methodology, for each device, the interstimulus interval varied from 2,000 to $10,000 \mathrm{msec}$, and the subjects did not receive feedback at the end of the test session.

Between testing sessions, the subjects had free time in the sleep laboratory, where they could eat, read, study, listen to music, watch television or videos, or play computer games. Regular balanced meals and snacks were provided. Throughout the study, the subjects were not permitted to consume caffeine, exercise, or nap.

Statistical analysis. Two commonly reported PVT parameters were analyzed for this report, each of which was derived from RT, the latency between the appearance of the stimulus and the buttonpush response. The first metric was the reciprocal of the mean RT (1/RT). Since previous studies had indicated systematic difference in absolute response speeds for the two devices (Lamond, Dawson, \& Roach, 2005; Thorne, Johnson, Redmond, et al., 2005), RTs were standardized ( $z$ scores) for each individual and then averaged. The second metric was mean number of lapses (RTs greater than $500 \mathrm{msec}$ ) per minute of testing.

Statistical analysis was performed with SPSS (Version 11.0.2, SPSS Inc., U.S.). Changes in each parameter across the experimental session and differences between the two tasks were evaluated using repeated measures ANOVA with two within-subjects factors (hours of wakefulness and task). In order to further investigate potential differences across the experimental period, a secondary analysis was performed in which data from each of the 3 days $(0900-2300)$ and 2 nights $(0100-0700)$ of testing were analyzed separately using repeated measures ANOVA with two within-subjects factors (hours of wakefulness and task). Huynh-Feldt corrections were applied to all repeated measures effects. A significance level of $p<.05$ was used for all the statistical analyses.

\section{Results}

Figure 1 displays data for mean response speed [(1/ mean RT) $\times 1,000]$ and mean number of lapses per minute as a function of task and hours of wakefulness. Analysis of response speed yielded a significant main effect of hours of wakefulness $[F(30,600)=42.8, p=.0001]$, in addition to a significant interaction $[F(30,600)=1.8, p=$ $.018]$. A main effect of task was not observed. For lapses (Figure 2), a significant main effect was found for both hours of wakefulness $[F(30,600)=28.6, p=.0001]$ and task $[F(1,20)=5.6, p=.029]$. In addition, a significant interaction $[F(30,600)=2.1, p=.004]$ was observed.

As is shown in Table 1, a separate analysis of data from Day 1 yielded a significant main effect of task for lapses, but not for response speed. A main effect of hours of wakefulness was not found for either parameter. For data collected during the first night, analysis yielded a signifi- 


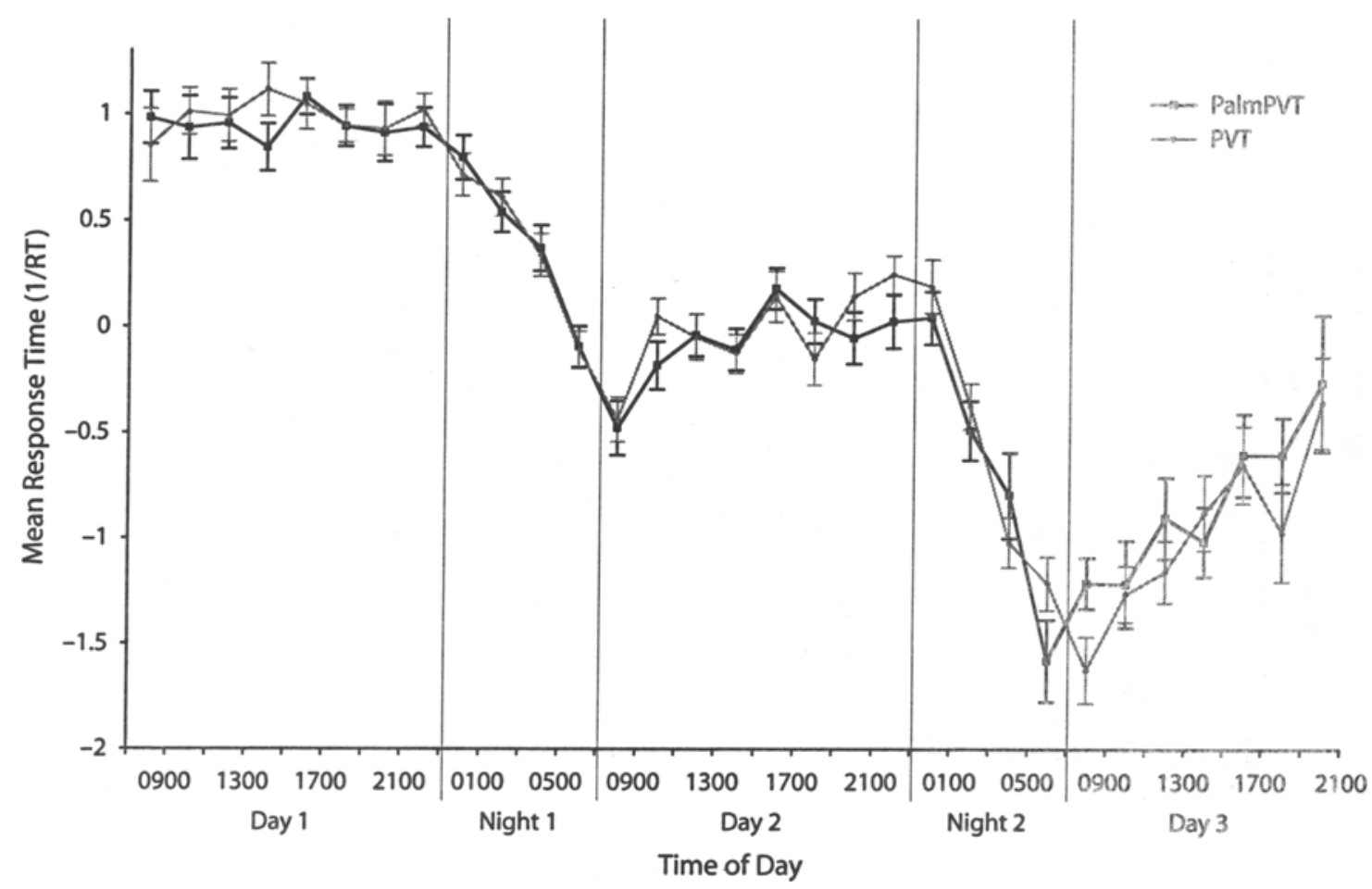

Figure 1. Mean ( \pm SEM) standardized response speed (1/RT) scores for the 10-min psychomotor vigilance taslz (PVT) and the 5-min PDA-PVT during 62 h of sustained wakefulness.

cant main effect of hours of wakefulness for both response speed and lapses. A main effect of task was not observed for either parameter, although it approached significance for lapses $(p=.063)$. Similarly, analyses indicated a significant main effect of hours of wakefulness for both parameters for both Day 2 and Night 2 data, in addition to a significant main effect of task for lapses. For data collected on Day 3, a significant main effect of both hours of wakefulness and task was observed for both response speed and lapses. No significant interaction effects were observed for either parameter when the data from each day/night were analyzed separately.

\section{Discussion}

The PDA-PVT has several advantages that recommend it for use as an assay of fatigue-related impairment in field settings. The device is small, inexpensive, and easy to use, and the task itself requires minimal aptitude and training. Feedback is optional, several individuals can share one device, the test can be self-administered, and using a 5-min version of the task reduces the impact of testing on an individual's day-to-day activities or, alternatively, increases the number of tests that can be performed within a given period. More important, two recent validation studies have demonstrated that the 5-min PDA-PVT is sensitive to the effects of moderate sleep loss and fatigue (e.g., 28-40 h of wakefulness), producing decrements comparable to those observed with the 10-min PVT-192. Thus, it is not surprising that the PDA-PVT is increasingly being used in field studies. The aim of the present study was to further validate the 5-min version of the palm PVT by assessing its sensitivity to greater levels of sleep loss and fatigue than had previously been studied.

The present study clearly demonstrates the sensitivity of the PDA-PVT to the effects of 2 nights of wakefulness. Consistent with the findings of previous studies documenting neurobehavioral deficits during extended periods of wakefulness (Lamond \& Dawson, 1999; Linde \& Bergstrom, 1992), response speed and lapses remained at a relatively stable level during the period that coincided with the normal waking day $(0-17 \mathrm{~h})$ for both the PDAPVT and the PVT-192. As hours of wakefulness increased, response speed significantly decreased and lapses significantly increased, with poorest performance on both tasks occurring after $47-49 \mathrm{~h}$ of wakefulness $(0800-1000 \mathrm{~h}$ of Day 3). Importantly, in line with previous findings, standardized response speed scores on the 5-min PDA-PVT closely tracked those of the PVT-192.

Although lapses followed the same general pattern for the two devices, there were statistically significant differences between the tasks. For most of the experimental period, significantly more lapses/minute were associated with the PDA-PVT. This is not particularly surprising given that, as has been noted, there are systematic differences in absolute response speeds between the two devices (approximately $50-100 \mathrm{msec}$ ), attributable to mechanical differences. Yet when the data are processed using the standard REACT analysis software program (Ambulatory Monitoring, Ardsley, NY), lapses are defined in the same way (i.e., RTs $>500 \mathrm{msec}$, an arbitrary criterion that was chosen because it is roughly equal to twice the baseline mean on the PVT-192). The disparity in lapses between 


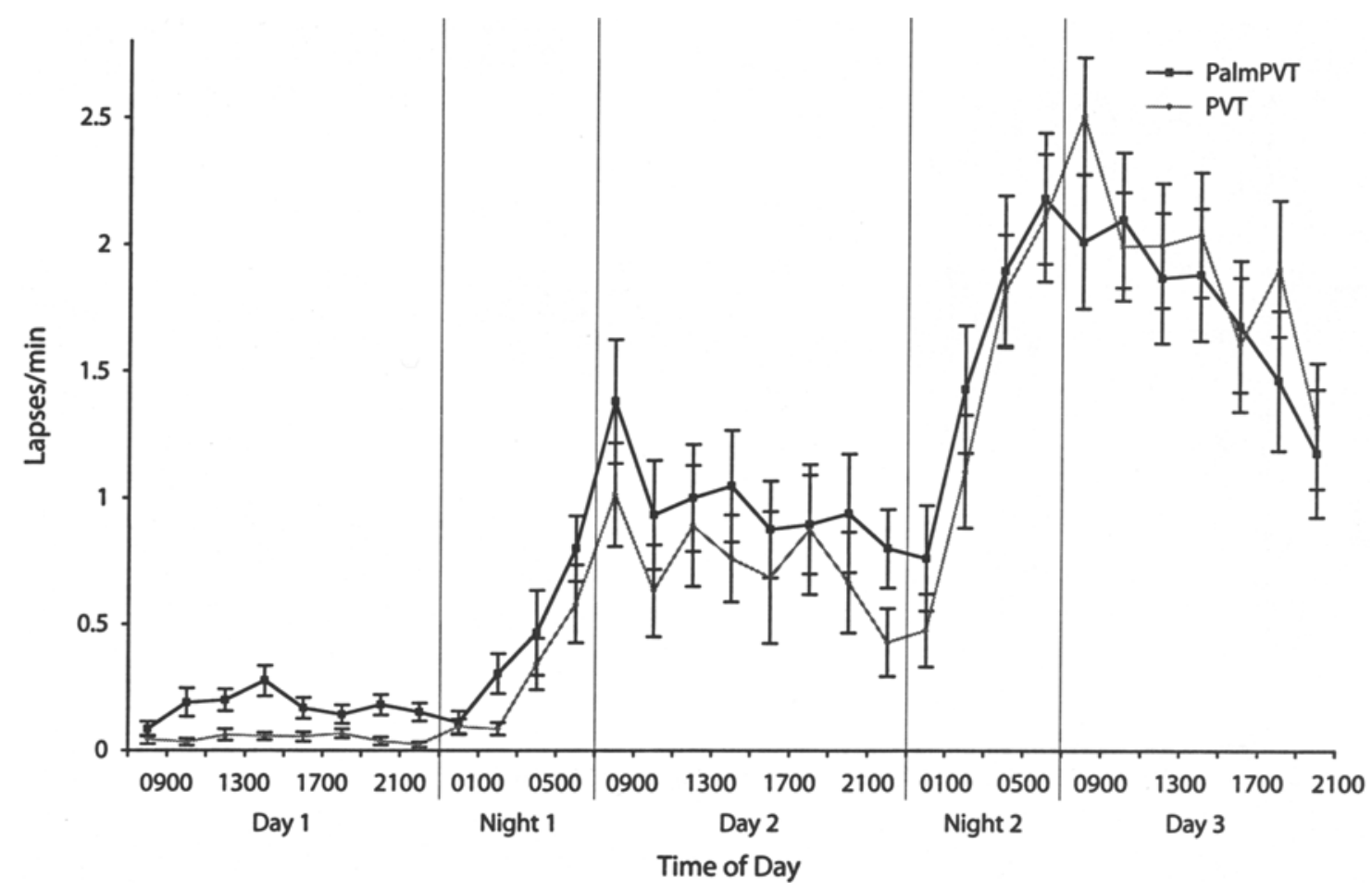

Figure 2. Mean ( $\pm S E M$ ) number of lapses/minute for the 10-min psychomotor vigilance task (PVT) and the 5-min PDA-PVT during 62 h of sustained wakefulness.

the two devices would effectively be removed if the criteria for lapses were redefined when data from the PDAPVT are analyzed (e.g., lapses $=$ RTs $>550 \mathrm{msec}$ ), to account for the systematic differences in response speed between the two devices.

Interestingly, analysis indicated that for both parameters, performance on the two tasks was, at least to some degree, differentially affected over the period of wakefulness. This contrasts somewhat with the findings of previous studies, in which the impairment associated with the 5-min PDA-PVT during moderate sleep loss closely par-

Table 1

Summary of ANOVA Results for Each Day and Night of the Experimental Period for Response Speed and Lapses

\begin{tabular}{|c|c|c|c|c|c|c|c|}
\hline \multirow[b]{2}{*}{ Phase } & \multicolumn{3}{|c|}{ Time } & \multicolumn{2}{|c|}{ Task } & \multicolumn{2}{|c|}{ Task $\times$ Time } \\
\hline & $d f$ & $F$ & $p$ & $F(1,20)$ & $p$ & $F^{*}$ & $p$ \\
\hline \multicolumn{8}{|l|}{ Day 1} \\
\hline 1/RT & 7,140 & 0.4 & n.s. & 1.4 & n.s. & 1.4 & n.s. \\
\hline Lapses & 7,140 & 1.7 & n.s. & 40.8 & .0001 & 1.5 & n.s. \\
\hline \multicolumn{8}{|l|}{ Night 1} \\
\hline 1/RT & 3,60 & 27.3 & .0001 & 0.1 & n.s. & 0.4 & n.s. \\
\hline Lapses & 3,60 & 10.0 & .0001 & 3.9 & n.s. ${ }^{\dagger}$ & 1.2 & n.s. \\
\hline \multicolumn{8}{|l|}{ Day 2} \\
\hline 1/RT & 7,140 & 5.8 & .0001 & 2.5 & n.s. & 2.1 & n.s. \\
\hline Lapses & 7,140 & 3.5 & .003 & 7.4 & .013 & 0.9 & n.s. \\
\hline \multicolumn{8}{|l|}{ Night 2} \\
\hline 1/RT & 3,60 & 34.7 & .0001 & 2.3 & n.s. & 2.2 & n.s. \\
\hline Lapses & 3,60 & 22.0 & .0001 & 4.8 & .041 & 0.5 & n.s. \\
\hline \multicolumn{8}{|l|}{ Day 3} \\
\hline I/RT & 6,120 & 7.9 & .0001 & 16.7 & .001 & 1.2 & n.s. \\
\hline Lapses & 6,120 & 4.8 & .001 & 6.7 & .017 & 1.3 & n.s. \\
\hline
\end{tabular}

Degrees of freedom ( $d f$ ) the same as for time. ${ }^{t} p=.063$. alleled that observed for the 10-min PVT-192 and, thus, no interaction effect was found. Closer inspection of the data indicated that when the level of sleep loss and fatigue became more severe, the 5-min PDA-PVT was less sensitive to the effects of the experimental manipulation than was the 10-min PVT-192. Thus, whereas the 5-min PDA-PVT was associated with more lapses than was the PVT-192 during the initial $48 \mathrm{~h}$ of wakefulness, the opposite was observed on the 3rd day of wakefulness (i.e., fewer lapses were associated with the PDA-PVT). Similarly, although PDA-PVT response speed closely tracked PVT-192 response speed initially, during the last day of wakefulness, PDA-PVT response speeds were slightly faster.

The observed differences in performance on the two tasks is not particularly surprising, given that past studies have consistently demonstrated that longer tasks, particularly monotonous ones, are more susceptible to performance lapsing during extended periods of wakefulness (Dinges \& Barone Kribbs, 1991). Indeed, it has been shown that significantly fewer lapses occur when the PVT-192 is shortened to only a 5-min duration (Lamond, Roach, \& Dawson, 2002; Roach, Dawson, \& Lamond, 2005), and thus, it is highly probable that the differences between the two devices was due solely to the difference in task duration. It is interesting, however, that the differential effect did not occur until the period of wakefulness had become more severe. Moreover, it is also worth noting that although there were differences in sensitivity between the tasks as the period of wakefulness became more severe, the differences were not necessarily functionally significant (i.e., $<0.5$ lapse/minute). As a final point, 
it should also be noted that using the same 2- to $10-\mathrm{sec}$ foreperiods for both a 5-and a 10-min task necessarily results in a smaller $N$ for the shorter task, thereby affecting the statistics reported (e.g., $F$ and $p$ values, SEMs, etc.). The default foreperiods for the PDA-PVT used in this study are 1-5 sec, which yields comparable $N$ s for the two tasks, and comparable results with a similarly configured PVT-192 (Thorne, Johnson, Kautz, \& Redmond, 2005).

The present study supports previous findings and indicates that the 5-min PDA-PVT is sensitive to the effects of sleep loss and fatigue. Although it is clear that the shorter task was not quite as sensitive to performance lapsing as was the 10-min task when 2- to 10-sec foreperiods were used for both, particularly when the levels of sleep loss and fatigue were more severe, this was most likely due solely to difference in task duration. Thus, the data suggest that the 5-min PDA-PVT is a valid alternative to the PVT-192 for assessing fatigue, particularly in situations in which (1) a shorter test is required, due to time restraints or to increase the number of tests that can be performed, (2) a small, convenient device (i.e., easy to transport and carry around) is required, and (3) a large number of devices are needed or multiple individuals need to share one device.

Clearly, as a controlled laboratory study, this validation was conducted using ideal conditions for testing. However, several of our field studies have demonstrated that the PDA-PVT is also suitable for use in the field, when conditions are often less than ideal. To date, we have successfully used this device in several work environments, including international flight operations, long-haul train travel, and mining operations (Baulk et al., 2007; Jay, Dawson, \& Lamond, 2005; Lamond et al., 2006; Petrilli et al., 2006). It is also important to note that although the PDAPYT may provide an indication of the fatigue levels associated with a roster, it is simply a sustained attention task, not a test of higher cognitive functions. As such, it does not necessarily indicate to what degree operational performance is affected by fatigue. Studies that are more operationally realistic, such as those using data loggers on trains (Dorrian, Hussey, \& Dawson, 2007) or realistic simulators (Roach, Dorrian, Fletcher, \& Dawson, 2001), would be better suited to answering questions such as these.

\section{AUTHOR NOTE}

The authors gratefully acknowledge David Thorne and Colonel Gregory Belenky for supplying us with the Walter Reed palm-held psychomotor vigilance task. We also thank the students who worked on various stages of the project and helped with data acquisition: Rochayne Kahl, Gemma Paech, Jessica Paterson, Candice Ryder, Helena lannos, Kirsty Willsmore, Nelson Yen, Chris Jones, Hannah Slattery, and Kimberley Pfeiffer. Correspondence concerning this article should be addressed to N. Lamond, Centre for Sleep Research, University of South Australia, Level 7 Playford Building, City East Campus, Frome Road, Adelaide, SA, 5000, Australia (e-mail: lamo0026@flinders.edu.au).

\section{REFERENCES}

BaulK, S. D., Kandelaars, K. J., Lamond, N., Roach, G. D., DawSON, D., \& FLETChER, A. (2007). Does variation in workload affect fatigue in a regular 12-hour shift system? Sleep \& Biological Rhythms, 5, 74-77.

Belenky, G., Wesensten, N. J., Thorne, D. R., Thomas, M. L., Sing,
H. C., ReDmond, D. P., ET AL. (2003). Patterns of performance degradation and restoration during sleep restriction and subsequent recovery: A sleep dose-response study. Journal of Sleep Research, 12, 1-12.

DAwson, D., \& REID, K. (1997). Fatigue, alcohol and performance impairment. Nature, 388, 235.

DiNGES, D. [F.] (1995). An overview of sleepiness and accidents. Journal of Sleep Research, 4(Suppl. 2), 4-14.

Dinges, D. F., \& Barone KrIBBS, N. (1991). Performing while experimentally sleepy: Effects of experimentally-induced sleepiness. In T. H. Monk (Ed.), Sleep, sleepiness and performance (pp. 97-128). New York: Wiley.

Dinges, D. F., Pack, F., Williams, K., Gillen, K. A., Powell, J. W., OTT, G. E., ET AL. (1997). Cumulative sleepiness, mood disturbance, and psychomotor vigilance performance decrements during a week of sleep restricted to 4-5 hours per night. Sleep, 20, 267-277.

Dinges, D. F., \& Powell, J. W. (1985). Microcomputer analyses of performance on a portable, simple visual RT task during sustained operations. Behavior Research Methods, Instruments, \& Computers, 17, 652-655.

DORRIAN, J., Hussey, F., \& Dawson, D. (2007). Train driving efficiency and safety: Examining the cost of fatigue. Journal of Sleep Research, 16, 1-11.

Dorrian, J., Lamond, N., van den Heuvel, C., Pincombe, J., Rogers, A. E., \& DAwson, D. (2006). A pilot study of the safety implications of Australian nurses' sleep and work hours. Chronobiology International, 23, 1149-1163.

Ferguson, S. A., DAwson, D., \& LAMOND, N. (2005). Study and analysis into fatigue coastal pilots. Adelaide: Centre for Sleep Research.

HaKKanen, J., \& Summala, H. (2000). Sleepiness at work among commercial truck drivers. Sleep, 23, 49-57.

House of Representatives Standing Committee on CommunicaTIONS, TRANSPORT, AND THE ARTs (2000). Beyond the midnight oil: An inquiry into managing fatigue in transport. Canberra: CanPrint Communications.

JAY, S. M., Dawson, D., \& LAMOND, N. (2005, September). Train drivers' fatigue and recovery during extended relay operations. Paper presented at the International Conference on Fatigue Management in Transportation Operations, Seattle.

JoHnSON, L. C. (1982). Sleep deprivation and performance. In W. B. Webb (Ed.), Biological rhythms, sleep, and performance (pp. 111-142). New York: Wiley.

Kammori, G. H., Johnson, D., Thorne, D., \& Belenky, G. L. (2005). Multiple caffeine doses maintain vigilance during early morning operations. Aviation, Space, \& Environmental Medicine, 76, 1046-1050.

Kullgore, W. D. S., KiLlgore, D. B., Ganesan, G., KRUGLER, A. L., \& KAMIMOR, G. H. (2006). Trait-anger enhances effects of caffeine on psychomotor vigilance performance. Perceptual \& Motor Skills, $103,883-886$.

LAmond, N., DARwENT, D., \& DAwson, D. (2005). Train drivers' sleep and alertness during short relay operations. Applied Ergonomics, 36, 313-318.

LAMOND, N., \& DAwSON, D. (1999). Quantifying the performance impairment associated with fatigue. Journal of Sleep Research, 8 , 255-262.

LAMOND, N., Dawson, D., \& RoACH, G. D. (2005). Fatigue assessment in the field: Validation of a hand-held electronic psychomotor vigilance task. Aviation, Space, \& Envinonmental Medicine, 76, 486-489.

Lamond, N., Petrilli, R. M., Dawson, D., \& Roach, G. D. (2006). Do short international layovers allow sufficient opportunity for pilots to recover? Chronobiology International, 23, 1285-1294.

Lamond, N., Roach, G. D., DarWent, D., Dorrian, J., Sletten, T., MCCULLOCH, K., ET AL. (2002). Australia railways shiftwork and workload study: Phase II final report. Adelaide: Centre for Sleep Research.

LAMOND, N., RoACH, G. D., \& DAwson, D. (2002). Is there an alternative to the 10-minute PVT for field studies? In I. G. N. Susila (Ed.), Kumpulan makalah ergonomi (pp. 512-521). Denpasar, Bali: Udayana University Press.

LAUber, J. K., \& KAYTEN, P. J. (1988). Sleepiness, circadian dysthythmia, and fatigue in transportation system accidents. Sleep, 11, 503-512. 
LiNDE, L., \& Bergstrom, M. (1992). The effect of one night without sleep on problem-solving and immediate recall. Psychological Research, 54, 127-136.

McLellan, T. M., Kamimori, G. H., Bell, D. G., SMith, I. F., JohNSON, D., \& BelENKY, G. (2005). Caffeine maintains vigilance and marksmanship in simulated urban operations with sleep deprivation. Aviation, Space, \& Environmental Medicine, 76, 39-45.

Mclellan, T. M., Kammori, G. H., Voss, D. M., Bell, D. G., Cole, K. G., \& Johnson, D. (2005). Caffeine maintains vigilance and improves run times in simulated night operations for special forces. Aviation, Space, \& Environmental Medicine, 76, 647-654.

Mitler, M., Carskadon, A. M., Czeisler, C. A., Dement, W. C. DiNGES, D. F., \& GraEBER, R. C. (1988). Catastrophes, sleep and public policy: Consensus report. Sleep, 11, 100-109.

Petrilli, R. M., Roach, G. D., Dawson, D., \& Lamond, N. (2006). The sleep, subjective fatigue, and sustained attention of commercial airline pilots during an international pattern. Chronobiology International, 23, 1357-1362.

RoACH, G. D., DAwson, D., \& LAMOND, N. (2005). Can a shorter PVT be used as a reasonable substitute for the 10-minute PVT? Shiftwort International Newsletter, 22, 129.

RoACH, G. D., DAWSON, D., \& LAMOND, N. (2006). Can a shorter psychomotor vigilance task be used as a reasonable substitute for the ten- minute psychomotor vigilance task? Chronobiology International, 23, $1379-1387$.

RoACh, G. D., Dorrian, J., Fletcher, A., \& Dawson, D. (2001). Comparing the effects of fatigue and alcohol consumption on locomotive engineers' performance in a rail simulator. Journal of Human Ergology, 30, 125-130.

RoACH, G. D., \& LAMOND, N. (2003). Fatigue risk management study. Qantas Airways Flight Ops Newsletter, 3, 42-43.

RosA, R. (1995). Extended workshifts and excessive fatigue. Journal of Sleep Research, 4(Suppl.), 51-56.

Smrth, L., Macdonald, I., Folkard, S., \& Tucker, P. (1998). Industrial shift systems. Applied Ergonomics, 29, 273-280.

Thorne, D. R., Johnson, D., Kautz, M., \& ReDMond, D. (2005). Further comparisons of a PDA-based PVT with the industry standard, using shortened test durations to improve compliance in field studies [Abstract]. Sleep, 28(Suppl.), A145.

Thorne, D. R., Johnson, D. E., Redmond, D. P., Sing, H. C., BelenKY, G., \& Shapiro, J. M. (2005). The Walter Reed palmheld psychomotor vigilance test. Behavior Research Methods, 37, 111-118.

(Manuscript received April 4, 2007; revision accepted for publication May $18,2007$. 